\title{
EXPERIMENTAL INVESTIGATION ON A CLOSED LOOP PULSATING HEAT PIPE IN HYPER-GRAVITY CONDITIONS
}

\author{
M. Mameli ${ }^{1 *}$, M. Manzoni ${ }^{1}$, L. Araneo ${ }^{2}$, S. Filippeschi ${ }^{3}$, M. Marengo ${ }^{1,4}$ \\ ${ }^{1}$ Università di Bergamo, Viale Marconi 5, 24044 Dalmine (BG), Italy \\ ${ }^{2}$ Politecnico di Milano, Dipartimento di Energia Via Lambruschini 4A, 20158 Milano, Italy \\ ${ }^{3}$ Università di Pisa, DESTEC, Largo Lazzarino 2, 56122 Pisa, Italy \\ ${ }^{4}$ School of Computing, Engineering and Mathematics, University of Brighton, Brighton BN2 4GJ, UK \\ ${ }^{*}$ Corresponding author
}

\begin{abstract}
A Closed Loop Pulsating Heat Pipe made of a copper tube (1.1mm/2mm I.D./O.D.) and filled with FC-72 has been tested on the Large Diameter Centrifuge (LDC) of the European Space Agency in Noordwijk at different hypergravity levels up to $20 \mathrm{~g}$, different heat input levels and different orientations with respect to the gravity direction (vertical bottom heated and horizontal position). Results shows that both in the horizontal and vertical orientation the device operation depends on the combined effect of gravity and heat input level. For the horizontal orientation, fluid stratification and the consequent thermal crisis occur at different acceleration levels depending also on the heat input power level and on the heating/gravity history. During the vertical operation the PHP thermal performance is slightly enhanced by the lower hyper-gravity levels (up to $3 \mathrm{~g}$ at $50 \mathrm{~W}$, up to $4 \mathrm{~g}$ at $70 \mathrm{~W}$ and up to $6 \mathrm{~g}$ at $100 \mathrm{~W}$ ) while two different local thermal crisis affect the PHP thermal behavior with the higher acceleration levels.
\end{abstract}

KEY WORDS: Two phase flow, Pulsating Heat Pipe, Hyper-gravity, Large Diameter Centrifuge.

\section{INTRODUCTION}

The present industry demand of high heat transfer capability coupled with relatively cheap component costs leads to the evolution of novel two-phase passive systems. The latent heat associated to evaporation and condensation is indeed a very efficient means of absorbing or releasing heat at relatively small temperature differences. The Closed Loop Pulsating Heat Pipe (CLPHP) is one of the most recent and promising twophase heat transfer devices, suitable for moderately high heat flux applications (up to $30 \mathrm{~W} / \mathrm{cm}^{2}$ ), that allows reduction of moving mechanical elements and could lead up to an efficient thermal control in space.

Patented in its most common assessment by Akachi [1,2], a CLPHP usually consists of a capillary diameter tube closed end-to-end, evacuated and then partially filled with a working fluid, which results as an alternation of vapor bubbles and liquid slugs inside the tube. When heat power is provided to the evaporator section, the thin liquid film, which surrounds each vapor plugs, evaporates, bubbles expand and push the adjacent fluid towards the condenser zone where heat is rejected to a cold source and condensation occurs within the vapor plugs nearby the inner wall surface.

In spite of the simple structure (e.g. no porous wick structures), the PHP working principles are complex: nowadays, there are no models able to fully describe and predict the actual behavior of a PHP both in transient and in pseudo-steady-state operation. Moreover a reliable database which contemplates a large variety of operating conditions (e.g. gravity modifications) cannot be easily found in literature. In particular, the behavior in hyper-gravity of a CLPHP has never been studied in detail, at least for a tube PHP, especially with regards to investigating the temperature trends and therefore the thermal resistance varying the heat flux. 
Except the on-going work by V. Ayel and C. Romestand, [3], there are only a few studies of PHP behavior under micro-gravity and hyper-gravity conditions, available in the literature.

$\mathrm{Gu}$ et al. [4,5] performed experiments in normal, hyper (2.5g) and microgravity ( $0.02 \mathrm{~g}$ on Falcon-20 flying parabolic trajectories) conditions on a Flat Plate Pulsating Heat Pipe (FPPHP) charged with R114, where square channels $(1.5 \mathrm{~mm})$ are engraved into an aluminum plate. One thermocouple has been used to characterize the heating and cooling sections and the device is always tested in two positions: vertical gravity assisted (Bottom Heated Mode, BHM), and vertical antigravity (Top Heated Mode, THM). They concluded that under normal and hyper-gravity conditions, the orientation affected the heat transfer performance. Moreover under reduced gravity, the heat pipes showed better operating and heat transport performance than when under normal and hyper-gravity conditions. However by taking a careful look at the results this last statement is evident only when comparing the normal gravity with the microgravity data obtained with the THM. Indeed it is expected that the occurrence of microgravity is beneficial when the device is working in the antigravity mode while it is not for the gravity assisted PHP.

Kiseev et al.[6] performed high-acceleration experiments on a $4.5 \mathrm{~m}$ diameter centrifuge table with a nonlooped acetone (60\% charge) device, consisting of 23 turns of $0.42 \mathrm{~m}$ long, $1.1 \mathrm{~mm}$ internal diameter (ID) stainless steel capillary. The evaporator and the adiabatic section were $120 \mathrm{~mm}$ long, while the condenser section was $180 \mathrm{~mm}$ long. Experiments were done for inclinations of $-90^{\circ}, 0^{\circ}$, and $+90^{\circ}$ with respect to the tangential direction. The so obtained data confirm that the acceleration influences the pressure and the corresponding temperature trends along such device: although dry-out was not observed, the evaporator increase its temperature from $160^{\circ} \mathrm{C}$ at $0 \mathrm{~g}$ to $200^{\circ} \mathrm{C}$ at $12 \mathrm{~g}$.

Ma et al.[7] investigate a three-dimensional flat-plate oscillating heat pipe (3D FP-OHP) under hyper-gravity loading with non-favorable evaporator positioning. The heat pipe $(3.0 \times 3.0 \times 0.254 \mathrm{~cm})$ was charged with acetone and tested with heat input of $95 \mathrm{~W}$ within a spin-table centrifuge. It was found that performances are near-independent of the investigated hyper-gravity loading up to $10 \mathrm{~g}$. Results show that the thermal conductivity of the investigated device remained near independent of gravity loading and slightly increase during $10 \mathrm{~g}$ loading; clearly, these findings suggest that device can operate in any orientation with respect to gravity and this demonstrate a robust thermal performance.

Van Es et al.[8] focused their experiments to study the basic working principle of the Flat Plate PHP consisting in a serpentine channel milled in an aluminum plate: the grooved plate is then covered by a thin top plate glued to the base plate. Finally the FSHP is partly filled with a working fluid, such as water or alcohol. The experiments were focused on fluid distribution, measurements of liquid and vapor slug oscillations, and assessment of the best working fluid and filling ratio. High-acceleration experiments were carried out on a rotating table, capable of generating $8.4 \mathrm{~g}$ on the FSHP center. The FSHP is heated by powering dissipating elements on the hot side; cooling was achieved by means of a Peltier element at the cold side; thermocouples were fixed on several locations on the FSHP. Two experiments were performed, one with a stainless steel prototype using ethanol as working fluid and one with an aluminum prototype filled with acetone. The experiments performed on the first prototype show an evaporator temperature first order increase to a stepwise boost in acceleration after few minutes of spinning at $4.4 \mathrm{~g}$. On the other hand, the second prototype was subjected to a high-g test, where the acceleration has been increased up to $8.4 \mathrm{~g}$. The maximum temperature difference over the FSHP was approximately $22.5^{\circ} \mathrm{C}$ and it remains constant with the acceleration increase underlining a stable operation of the FSHP.

Delil at al.[9-12] discuss the thermal-gravitational modeling and scaling of two-phase heat transport systems for spacecraft applications up to $12 \mathrm{~g}$. This investigation follows some previous works carried out at NLR on the same subject. They dealt with mechanically and capillary pumped two-phase loops. The activities pertained to pure geometric, pure fluid to fluid, or hybrid scaling between a prototype system and a model at the same gravity level, and between a prototype in gravity modified condition and a model on normal g-level condition. However, no comparison with experimental results are shown.

The objective of the present work is to study the combined effect of heat input and hyper-gravity level either on the Closed Loop Pulsating Heat Pipe (CLPHP) thermal performance, either on the local fluid pressure fluctuations in transient and pseudo-steady-state conditions. Indeed a $2 \mathrm{D}$ serpentine CLPHP made of a copper tube (O.D./I.D. $=2.0 / 1.1 \mathrm{~mm})$ charged with FC-72 (50\% filling ratio) and equipped with 13 "T" type thermocouples ( 9 in the evaporator and 4 in the condenser) have been tested at different heat loads (50, 70 and $100 \mathrm{~W}$ ), orientations (vertical bottom heated and horizontal), and gravity levels (from ground gravity up to 20g) on the European Space Agency (ESA) Large Diameter Centrifuge (LDC) in Noordwijk (NL) [13]. 


\section{EXPERIMENTS}

\subsection{Experimental apparatus}

The geometric characteristics of the PHP are briefly described in Fig. 1. The basic PHP structure consists of a copper tube having an internal diameter of $1.1 \mathrm{~mm}$ and external diameter of $2.0 \mathrm{~mm}$ folded so as to obtain 32 parallel channels and $16 U$ bends in the evaporator zone and then closed end to end by means of two $T$ joints. A pressure transducer (Kulite ${ }^{\circledR}$, ETL/T 312, 1.2bar A) is located at the condenser section. The PHP is equipped with 14 "T" type thermocouples (wire diameter $0.127 \mathrm{~mm}$, accuracy of $\pm 0.1^{\circ} \mathrm{C}$ after calibration): 9 are tin soldered on the external tube surface in the evaporator and 4 in the condenser in order to maximize the thermal contact; the last one measures the environmental temperature. The test cell geometry as well as the thermocouples location are shown in Fig. 1.

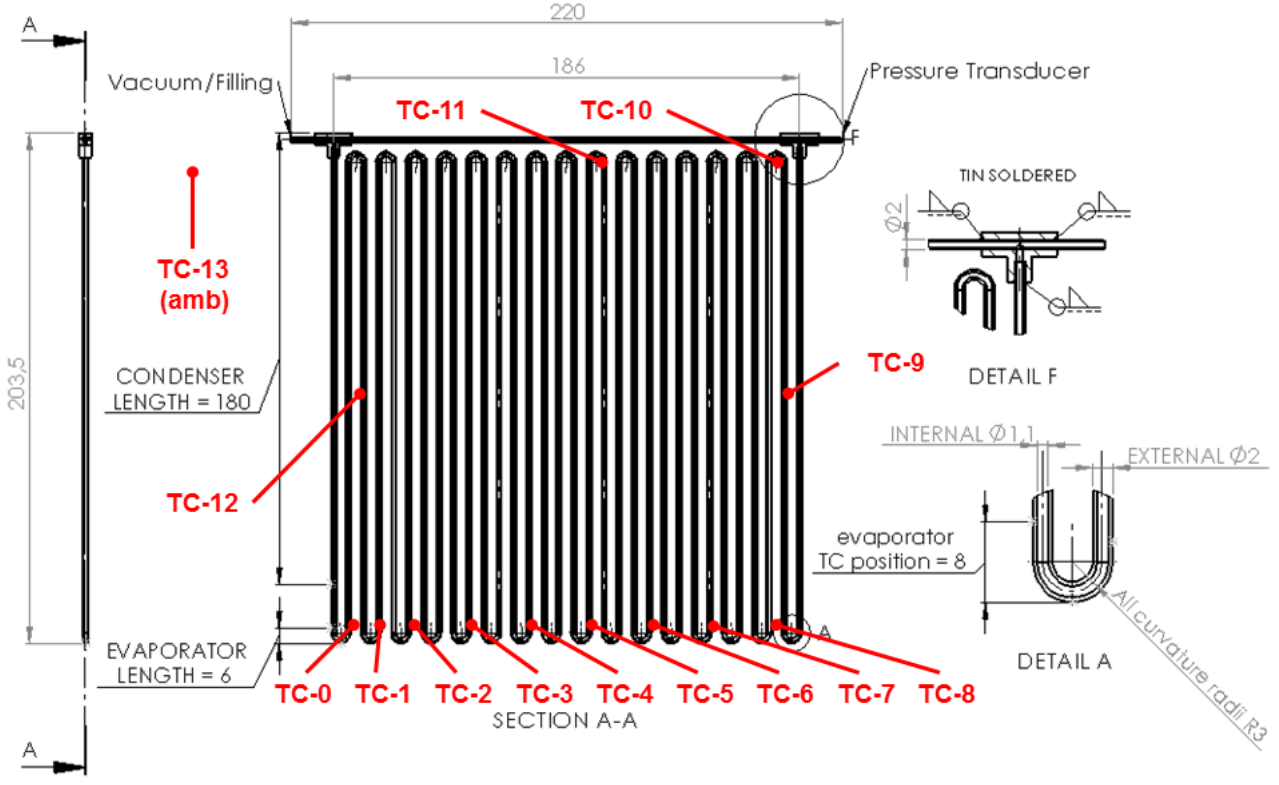

Fig. 1: Technical sketch of the PHP.

While evacuating the PHP by means of a ultra-high vacuum system (Varian ${ }^{\circledR}$ DS42 and TV81-T) down to $3.0^{*} 10^{-4} \mathrm{~Pa}$, the working fluid (FC-72) is degased in a secondary loop by continuous boiling and vacuuming cycles. Finally the PHP is filled with a volumetric ratio $0.5 \pm 0.03$ and permanently sealed by means of tin soldering. The incondensable gas content, less than 6PPM, is estimated by measuring the difference between the actual fluid pressure inside the PHP and its saturation pressure at environmental temperature.

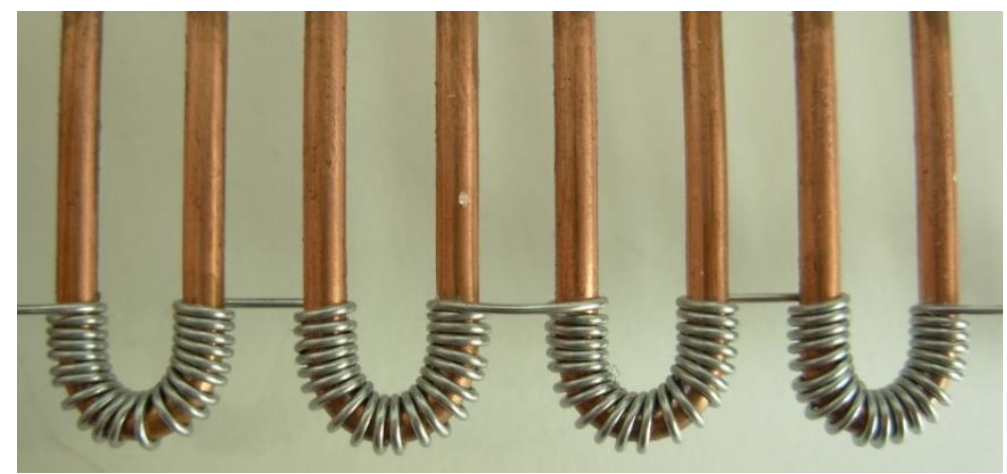

Fig. 2: Heating wire wrapped on evaporator section.

The PHP heater consists of 4 electric resistors connected in parallel (Thermocoax ${ }^{\circledR}$ Single core $1 \mathrm{Nc} A c$ ) with a total length of $3 \mathrm{~m}, 0.5 \mathrm{~mm}$ external diameter, and total electrical resistance $36 \Omega$. The wire is wrapped around the evaporator bends, as shown in Fig. 2. The PHP evaporator and the heater are protected by a Polyetheretherketone (PEEK) cover. Anyway the heater temperature is limited to $150^{\circ} \mathrm{C}$ by two thermo-fuses 
(DMP 11MP 150H 046E). Electric power, up to $100 \mathrm{~W}$, is provided by a power supply (GWInstek $\left.{ }^{\circledR} 3610 \mathrm{~A}\right)$ corresponding to a radial heat flux up to $12 \mathrm{~W} / \mathrm{cm}^{2} \pm 0.3$. Thermal contact is obtained by heat sink compound.
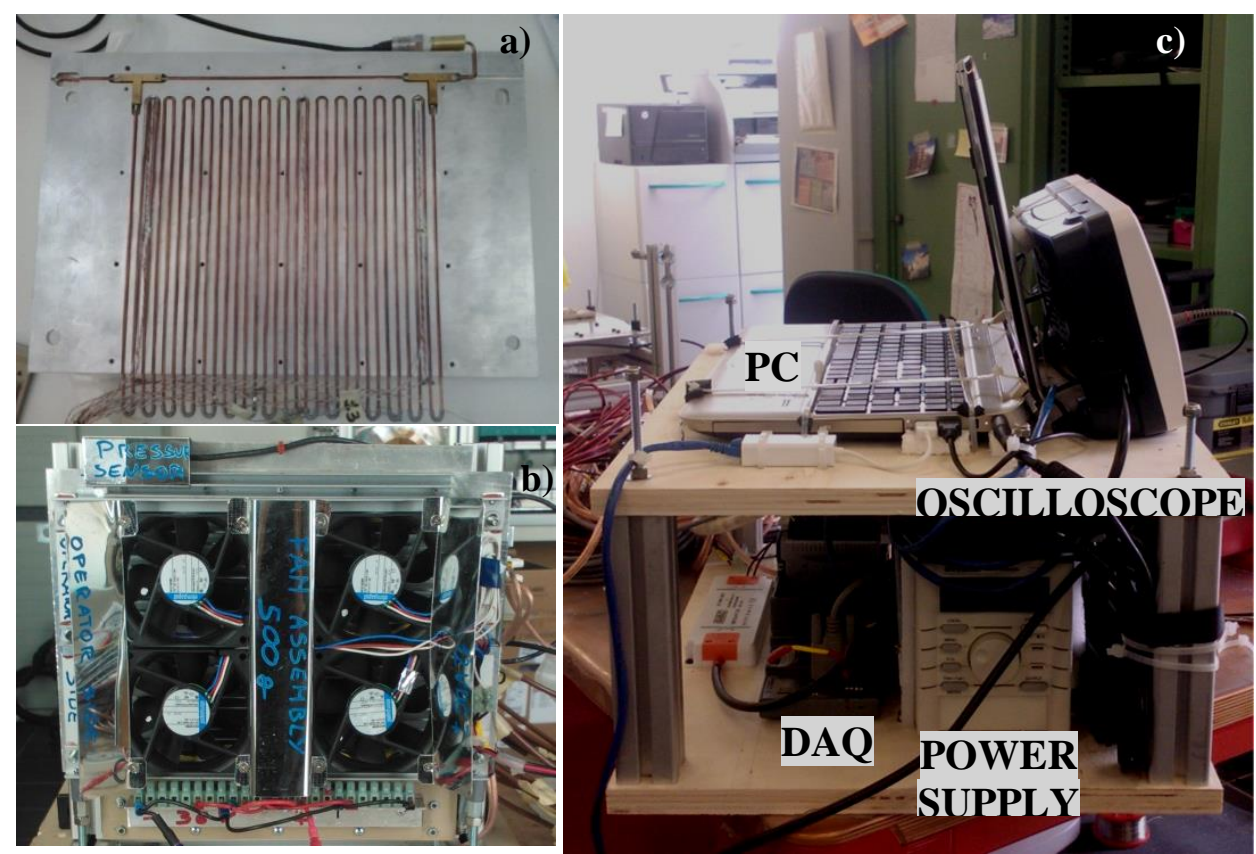

Fig. 3 a) PHP condenser section embedded into the aluminum heat sink; b) Air fan system; c) Peripheral facilities.

The condenser section is $180 \mathrm{~mm}$ long and it is cooled by means of a heat sink and air fans system. Circular cross section channels are milled on the back surface of the aluminum heat sink so as to host the copper tubes. The PHP condenser is embedded into the heat sink (Fig. 3a) and fixed with an aluminum back plate. Thermal contact is obtained by heat sink compound. Four air fans (ebmpapst.co.uk ${ }^{\circledR} 8412 \mathrm{~N} / 2 \mathrm{GH}-214$ ) are located on the heat sink fins in suction mode as shown in Fig. 3b. The effect of the LDC acceleration on the fans rotation speed is shown in Fig. 4. The PHP in the vertical position undergoes a negligible fan speed reduction, while in the horizontal position this reduction increases with the acceleration level. The horizontal tests have been performed up to $20 \mathrm{~g}$ where the reduction $(-12 \%)$ is critical for the cooling characteristics. | Therefore only the results up to $10 \mathrm{~g}$ will be considered for the horizontal position.

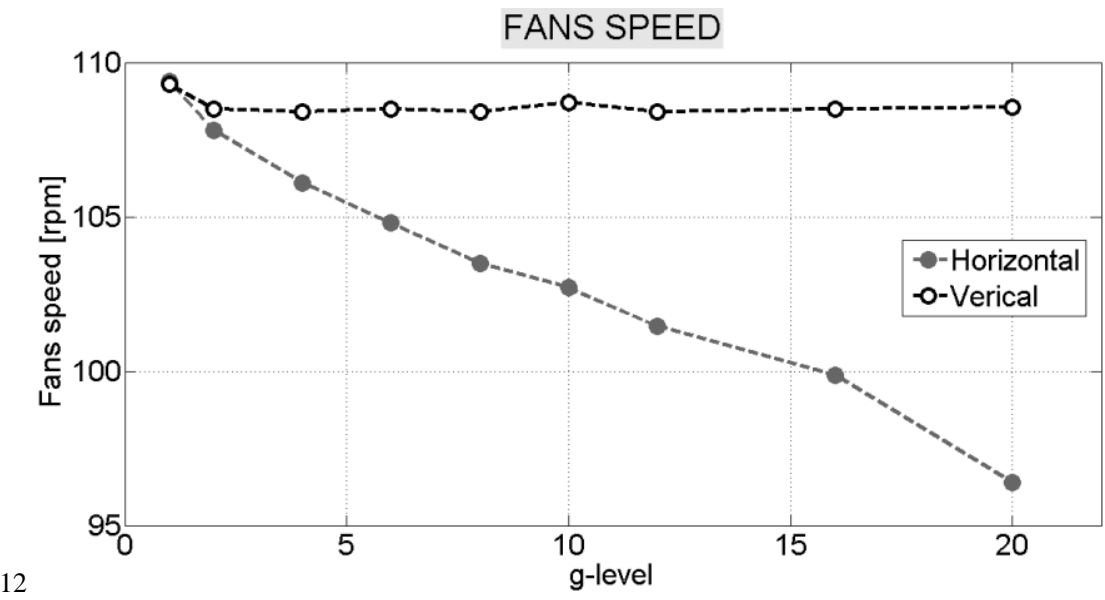

Fig. 4: Effect of the gravity level on the air fan speed. 
The PHP is also equipped with a g-sensor (Analog Devices ${ }^{\circledR}$ ADXL326) located on one side of the heat sink. The thermocouples, the pressure transducer, the g-sensor and the power supply are connected to a data acquisition system (NI-cRIO-9073, NI-9214 ${ }^{\circledR}$ ) and all signals are recorded at $16 \mathrm{~Hz}$.

The test-cell (PHP, thermocouples, pressure transducer, g sensor, heating and cooling system) is positioned on a beam structure by means of four anti-vibration bushes. Then, the structure may be fixed on the test rig plate with two different orientations: PHP vertical bottom heated, PHP horizontal as shown in Fig. 5.
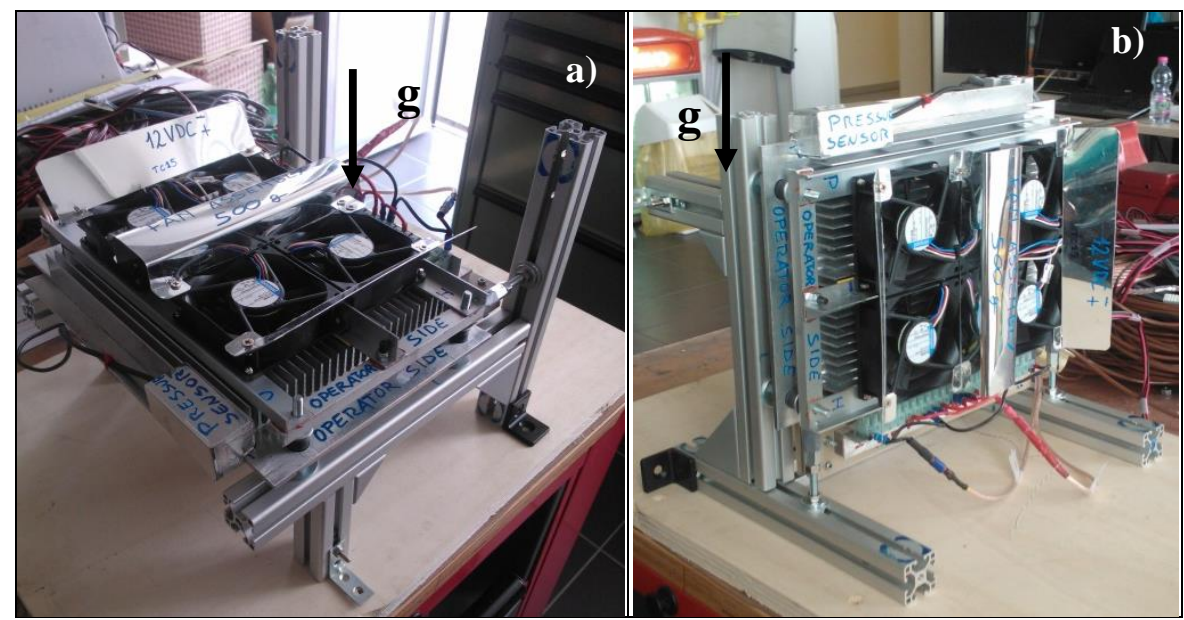

Fig. 5: Pulsating Heat Pipe position with respect to the gravity direction; a) Horizontal; b) Vertical.

Only the PHP test cell (PHP tube, heater, condenser and sensors) is located in the gondola (Fig. 6) while all the peripheral facilities (power supply, data logger, laptop and oscilloscope shown in Fig. 3c) are located in the LDC hub in order to avoid hyper-gravity on the electronic equipment. Shielded wires $(6.5 \mathrm{~m}$ long) then pass through the LDC branch and connect the test cell to the hardware in the hub.

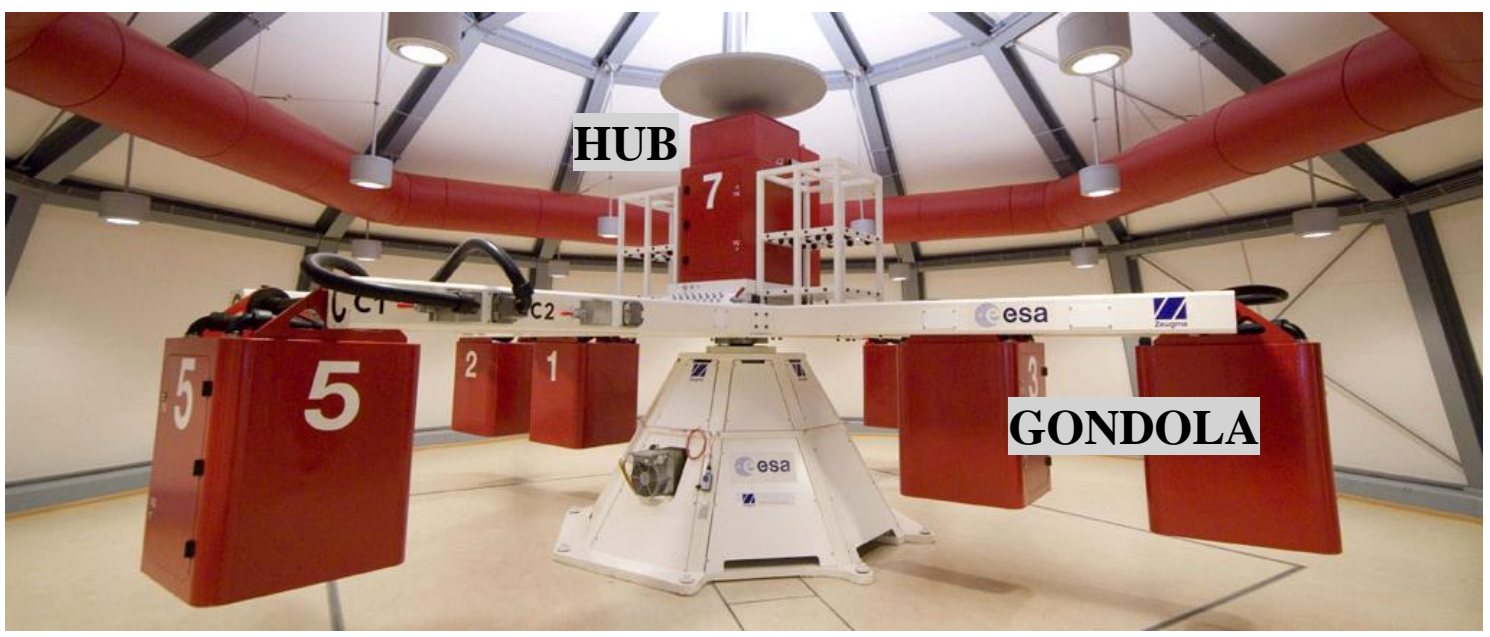

Fig. 6: Large Diameter Centrifuge: hub and gondolas.

There are no mechanical connections between the gondola floor and the PHP support since the test rig is kept still and stable thanks to the gravity modified acceleration; the LDC is, indeed, a passive swing out system and the gondola will orient such that the gravity vector is always perpendicular to its floor.

\subsection{Experimental Procedure}

The list of both ground and hyper-gravity experiments is shown in Table 1 . The device has been tested in two orientations with respect to the gravity direction (vertical bottom heated, horizontal) as shown in Fig. 5, different heating power levels $(50,70,100 \mathrm{~W})$ and different gravity levels $(1,2,4,6,10,12,16,20 \mathrm{~g})$. 
Table 1: List of the performed experiments.

\begin{tabular}{c|c|c|c|c|c}
\hline $\mathrm{N}^{\circ}$ Test & Location & Orientation & Heat Input $[\mathrm{W}]$ & g-level & Repeated \\
\hline 1 & Ground & Horizontal & $50 \rightarrow 70 \rightarrow 100$ & 1 & $\mathrm{Y}$ \\
\hline 2 & Ground & Vertical & $50 \rightarrow 70 \rightarrow 100$ & 1 & $\mathrm{Y}$ \\
\hline 3 & LDC & Horizontal & $2 \mathrm{x}(50 \rightarrow 70 \rightarrow 100 \rightarrow 70 \rightarrow 50)$ & $2 \rightarrow 4 \rightarrow 6 \rightarrow 10$ & $\mathrm{Y}$ \\
4 & LDC & Horizontal & 100 & $1 \rightarrow 2 \rightarrow 4 \rightarrow 6 \rightarrow 8 \rightarrow 10$ & $\mathrm{Y}$ \\
\hline 5 & LDC & Horizontal & 70 & $1 \rightarrow 4 \rightarrow 8 \rightarrow 10 \rightarrow 12 \rightarrow 16 \rightarrow 20$ & $\mathrm{~N}$ \\
6 & LDC & Horizontal & 50 & $1 \rightarrow 4 \rightarrow 6 \rightarrow 8 \rightarrow 10$ & $\mathrm{~N}$ \\
\hline 7 & LDC & Vertical & $50 \rightarrow 70 \rightarrow 100$ & 2 & $\mathrm{Y}$ \\
\hline 8 & LDC & Vertical & $50 \rightarrow 70 \rightarrow 100$ & 4 & $\mathrm{Y}$ \\
\hline 9 & LDC & Vertical & $50 \rightarrow 70 \rightarrow 100$ & 6 & $\mathrm{Y}$ \\
\hline 10 & LDC & Vertical & $50 \rightarrow 70 \rightarrow 100$ & 10 & $\mathrm{Y}$ \\
11 & LDC & Vertical & 100 & $1 \rightarrow 4 \rightarrow 8 \rightarrow 10 \rightarrow 12 \rightarrow 16 \rightarrow 20$ & $\mathrm{Y}$ \\
\hline 12 & LDC & Vertical & 70 & $1 \rightarrow 4 \rightarrow 5 \rightarrow 6 \rightarrow 7 \rightarrow 8 \rightarrow 10 \rightarrow 12$ & $\mathrm{~N}$ \\
\hline 13 & LDC & Vertical & 50 & $1 \rightarrow 2 \rightarrow 3 \rightarrow 4 \rightarrow 5 \rightarrow 6 \rightarrow 8 \rightarrow 10$ & $\mathrm{~N}$ \\
\hline
\end{tabular}

Every experiment has been carried out with the following procedure: first, the PHP test rig is arranged in vertical (bottom heat mode) or horizontal configuration and, if hyper-gravity is required, the PHP is placed inside an LDC gondola. Then the gondola is closed and the gravity vs. time profile is set. Afterwards, the initial heat input level is set and the experiment is powered up at normal gravity (1g) to warm-up the PHP for at least 8 minutes; even though every test is performed with a peculiar gravity and heat power history, every heat input or gravity level step is then kept for 8 minutes (the system is able to reach the pseudo-steady-state, which means all temperature signals show an average value constant in time, in about 180s). Finally the heating power supply is switched off and a cool down phase (15 minutes) is performed at normal g-level so that all temperatures reach the room conditions. Repeatability test have been performed for almost every testing condition.

\section{RESULTS}

Results are presented mainly in terms of temperature and pressure time evolutions. The tube wall temperature trends, both in the evaporator zone (reddish colors), condenser zone (bluish colors), and environment (green), are shown together with the heat input level on the secondary y-axis in the above part while the local fluid pressure in the condenser together with the heat input level on the secondary y-axis is shown below. In case of centrifugal test, the gravity acceleration is reported directly over the plot line.

\subsection{Ground test}

Ground test have been performed in the vertical and horizontal position increasing the heat input from 50W to $100 \mathrm{~W}$ with an intermediate step at $70 \mathrm{~W}$. The starting heat input level is kept for 16 minutes while the following are kept for 8 minutes each as shown in Fig. 7. Vertical (Fig. 7a) and horizontal (Fig. 7a) orientations show very different behaviors confirming that, in a perfect 2D layout with a relatively high number of channels, gravity still plays an important effect on the PHP thermal behavior. Indeed in the horizontal working mode the fluid motion is not assisted by gravity anymore, oscillations are less frequent the heat transfer rate is less efficient too and consequently temperatures in the evaporator set to a higher level with respect to the vertical position. After a start-up period of about 180 s, temperatures and pressure are able to reach a regular oscillating regime. In the vertical case the evaporator temperatures set in a $10^{\circ} \mathrm{C}$ range which is narrower than the horizontal one (up to $30^{\circ} \mathrm{C}$ ). The vertical operation shows an interesting feature at the higher heat input level (100W): some channels (only one in this case) undergo a sudden thermal crisis probably due to fluid motion dampening or local dry-out; its temperature is more than $30^{\circ} \mathrm{C}$ higher than all the other measured in the evaporator. 


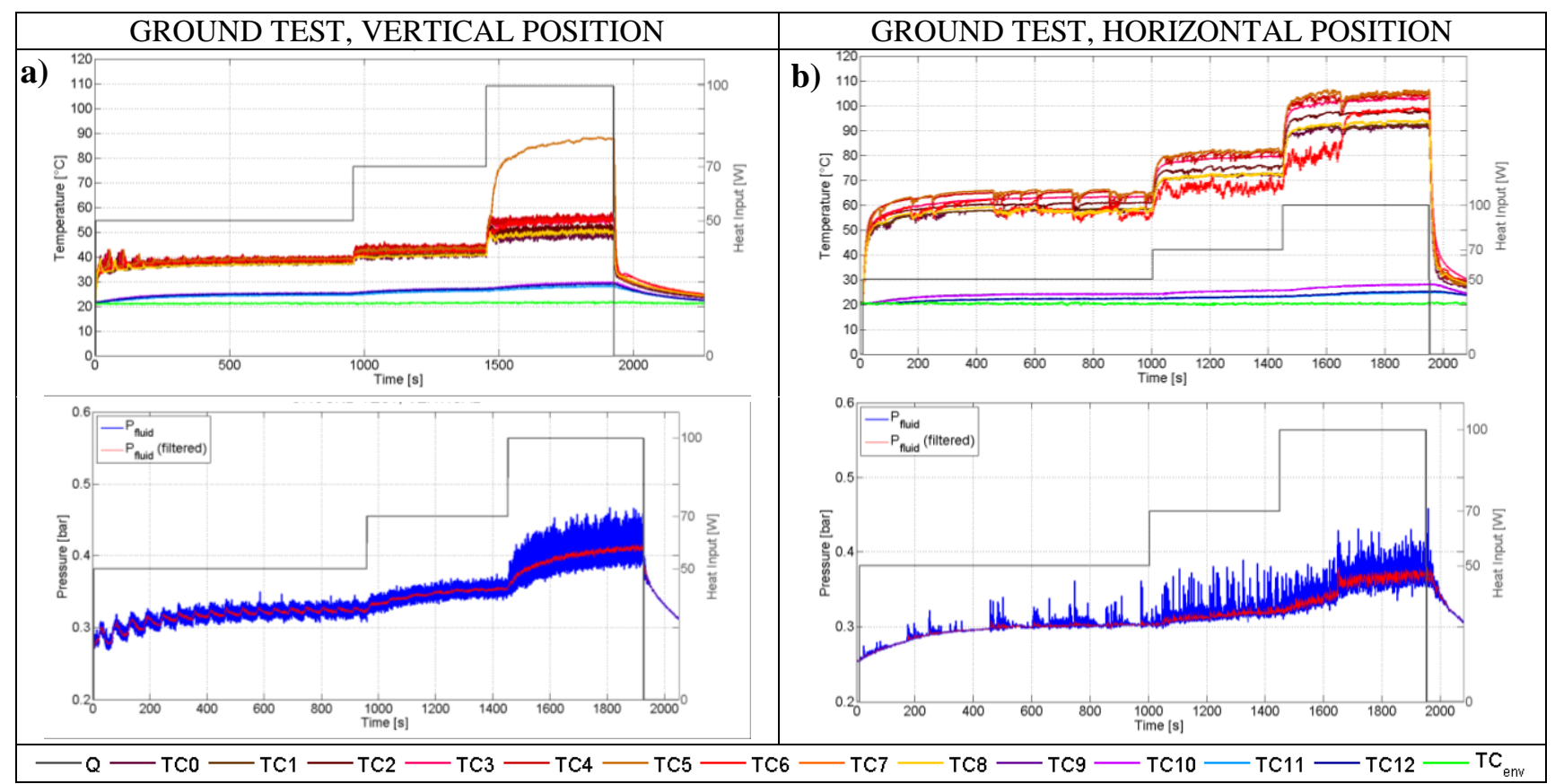

Fig. 5: Ground experiments, tube wall temperatures and local fluid pressure in the vertical position a) and horizontal position $b$ ).

\subsection{Hyper-gravity test}

The Large Diameter Centrifuge allows reaching accelerations up to 20 times the terrestrial gravity maximum. For the first time in literature, a tube CLPHP is tested in horizontal and vertical position at such high acceleration levels. The combined effect of heat input and gravity levels on the PHP thermal behavior is described here below.

\section{Horizontal position}

In this case the gravity vector is perpendicular to the flow path, thus the flow motion is not directly affected by the increasing acceleration. However the ratio between the buoyancy forces and the surface tension forces acting on the liquid, changes with gravity and consequently the flow pattern may be altered. Indeed, in order to have an initial slug-plug distribution, the fluid confinement criterion [14] $B o \leq 4$ should be satisfied (Bond Number is defined by $B o=g\left(\rho_{l}-\rho_{v}\right) D^{2} / \sigma$ where $\mathrm{g}$ is the gravity acceleration, $\rho_{l}-\rho_{v}$ is the difference between the liquid and vapor density at saturated conditions, $\mathrm{D}$ is the tube internal diameter and $\sigma$ is the surface tension). The critical diameter obtained by the previous criterion is plotted in Fig. 8 as a function of temperature at different acceleration levels.

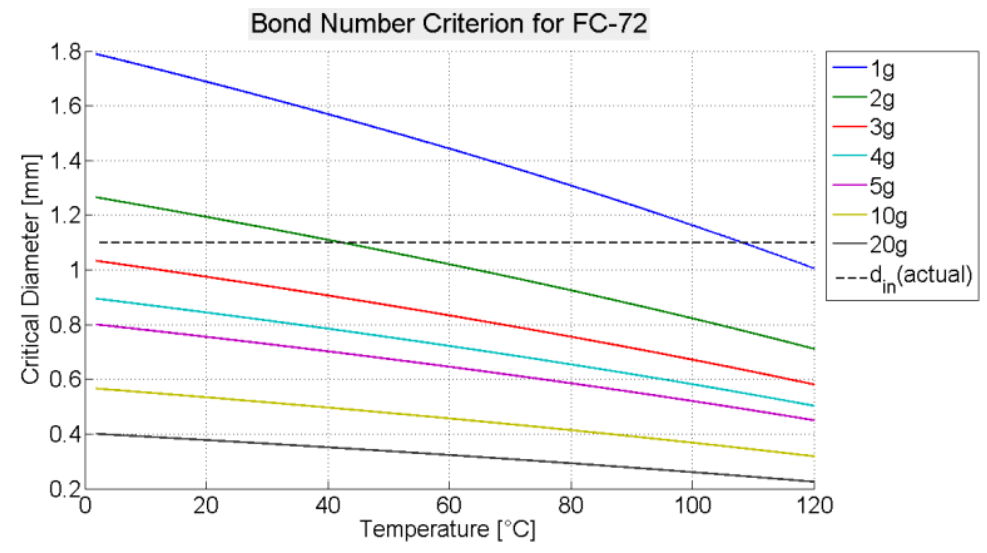

Fig. 6: Critical internal diameter as function of temperature at different acceleration levels. 
The actual CLPHP internal tube diameter is plotted with a dashed line: if the critical diameter is less than the actual diameter, the fluid may not be in the confined region anymore and stratification may occur. Looking at Fig. 8, theoretically, this transition should occur between 2 and 3g.

Since such PHP configuration does not allow any fluid visualization, it is possible to argue about the flow transition only in terms of thermal response and local fluid pressure fluctuations. Indeed it is here assumed that the flow regime is altered when all the temperatures in the evaporator together with the local pressure in the condenser react instantaneously to a change in the acceleration level.

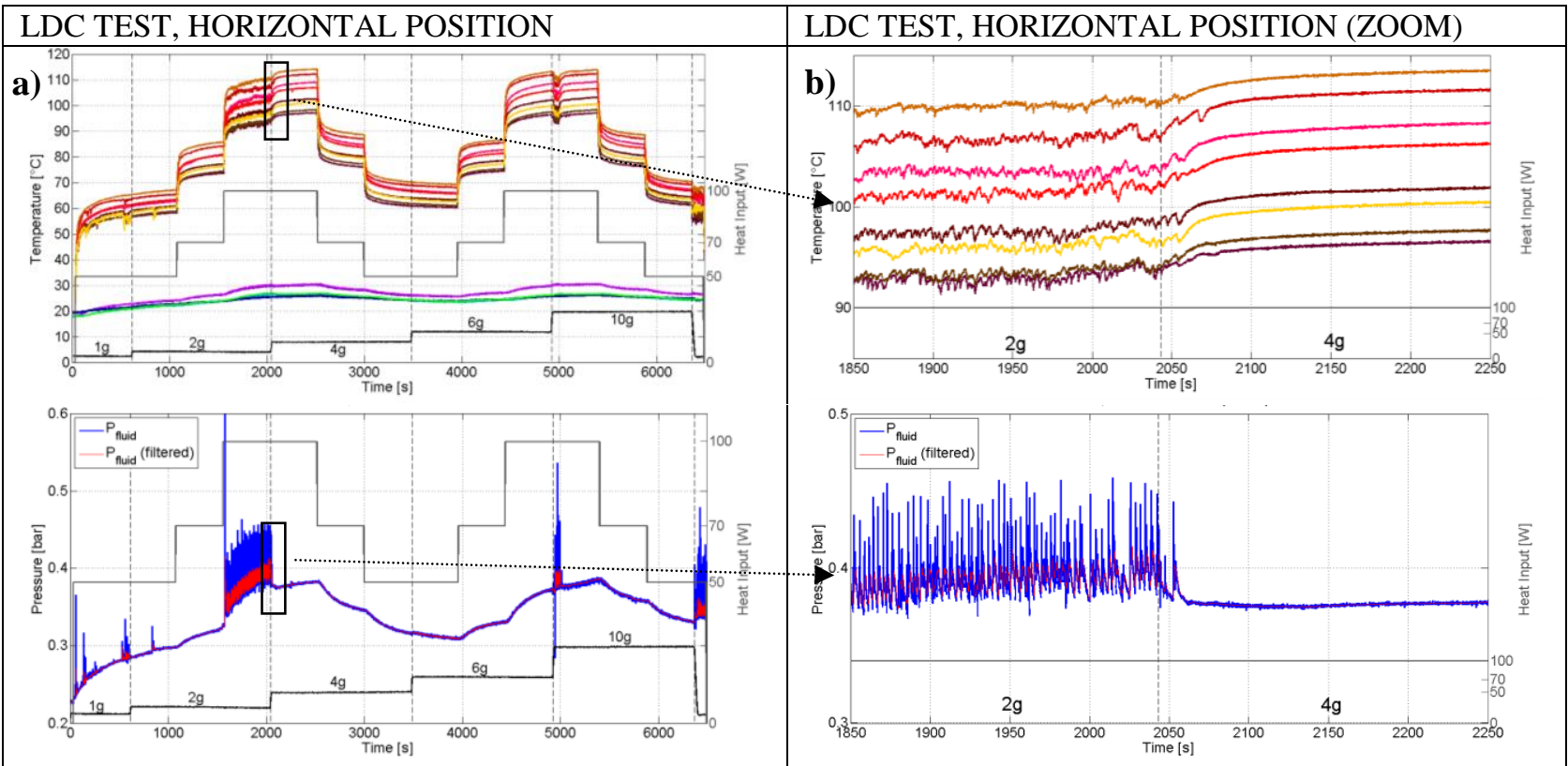

Fig. 7: Large Diameter Centrifuge experiments, a) tube wall temperatures and local fluid pressure in the horizontal position at different heat input and gravity levels; b) zoom on the transition between $2 \mathrm{~g}$ and $4 \mathrm{~g}$.

The first test in the horizontal position is carried out by changing both the heat input and the gravity level. At $50 \mathrm{~W}$ and $1 \mathrm{~g}$ the device is operating in a start-stop mode and this is clearly visible from the very poor pressure and temperature fluctuation in Fig. 9a. When the gravity level is increased to $2 \mathrm{~g}$ only one oscillating event is recognizable at $50 \mathrm{~W}$. Keeping the spin at $2 \mathrm{~g}$, the heat input level is increased to $70 \mathrm{~W}$ and the fluid motion seems completely damped out: there are no signal oscillations both in the fluid pressure and wall temperatures. Only when the heat input is set to $100 \mathrm{~W}$, the fluid pressure shows a higher peek, oscillation is reactivated and kept stable till the next increase in the gravity level. Indeed when the spin is increased to $4 \mathrm{~g}$ the device stops working in a few seconds: fluid pressure as well as the wall temperatures do not oscillate and thermal performance slightly decreases as shown in Fig. 7b. Afterwards, the heat input level is decreased to $70 \mathrm{~W}$ and then $50 \mathrm{~W}$ at $4 \mathrm{~g}$ but no reactivation occurred, as expected.

For the next duty cycle, gravity is increased to $6 \mathrm{~g}$ and, again, the heat input level is increased from $50 \mathrm{~W}$ to $100 \mathrm{~W}$. Only a sudden pressure peek is recorded at $100 \mathrm{~W}$ during the transition from 6 to $10 \mathrm{~g}$ but, contrary to the $2 \mathrm{~g}$ case, it is only a local event, not sufficient to restart the device operation. No operation is detected afterwards in hyper-gravity but, when the LDC stops and the gravity level gets back to $1 \mathrm{~g}$, the PHP recovers a vigorous temperature and pressure oscillation. The experiment has been repeated and no substantial differences arose. 

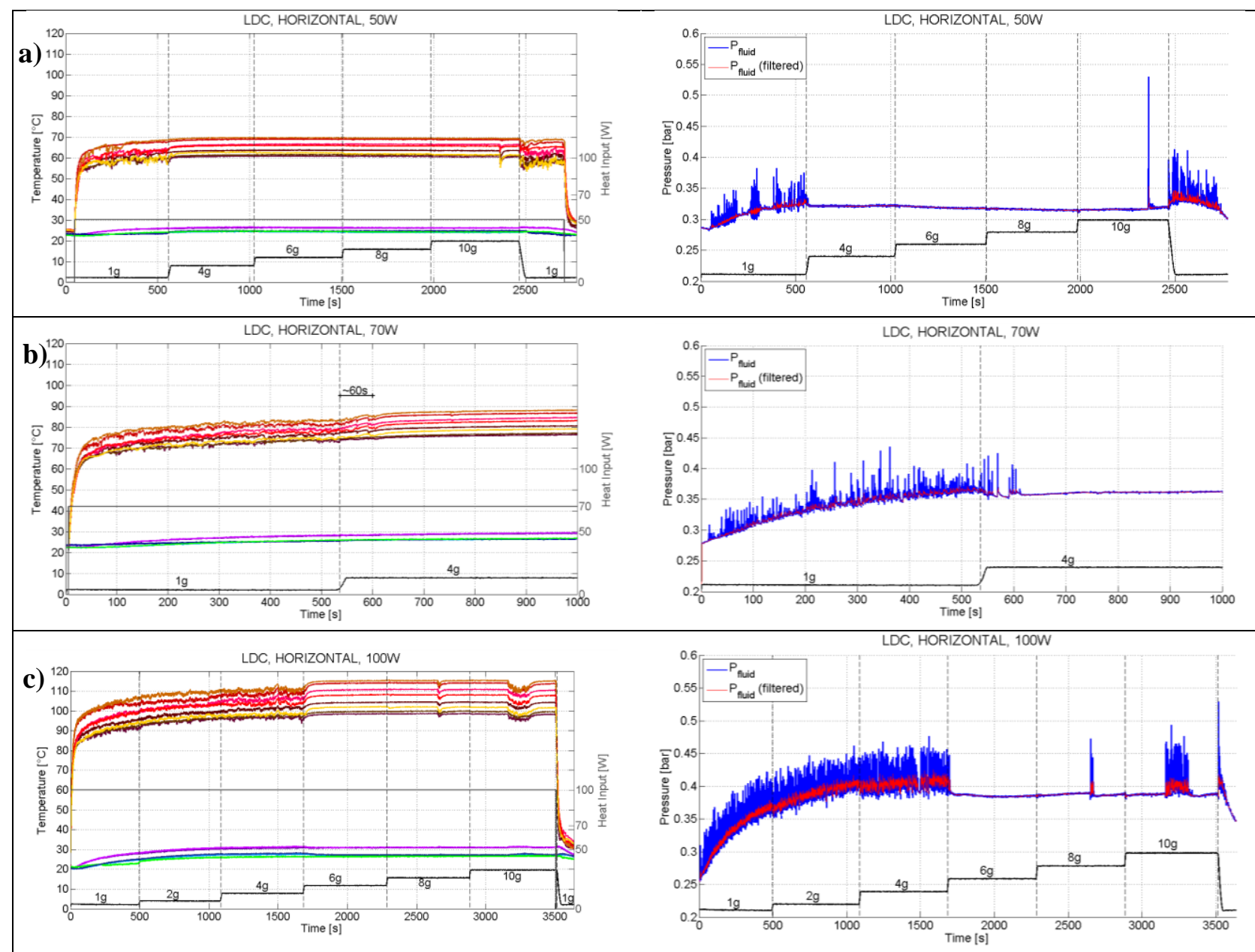

Fig. 8: Large Diameter Centrifuge experiments tube wall temperatures and local fluid pressure in the horizontal position and different gravity levels at, a) $50 \mathrm{~W}$; b) $70 \mathrm{~W}$ and c) at $100 \mathrm{~W}$.

In order to verify the hysteresis effect linked to the heating/gravity history, test are performed at increasing acceleration levels keeping constant the heat input at the evaporator. As expected from the results shown in Fig. 9, both test at 50 and $70 \mathrm{~W}$ show that the PHP stops working when gravity changes from 1 to $4 \mathrm{~g}$ but the switching off is almost instantaneous at $50 \mathrm{~W}$ (Fig. 10 a) while almost one minute is needed at $70 \mathrm{~W}$ (Fig. 10b). Differently, if the heat input level is kept at $100 \mathrm{~W}$ since the very beginning, the PHP is able to maintain a stable operation up to $4 \mathrm{~g}$ showing, by comparison with Fig. 9, a dependence on the heating/gravity history. Local fluid settlements occur at $8 \mathrm{~g}$ and at $10 \mathrm{~g}$ but, as in the previous experiments, the local event is not sufficient to restart the device operations. Hyper-gravity test have been performed up to $20 \mathrm{~g}$ at $70 \mathrm{~W}$ but no results are shown only up to $4 \mathrm{~g}$ because no PHP operation have been recorded above this gravity level.

\section{Vertical position}

When the PHP is oriented in the vertical bottom heated position, the acceleration vector is parallel to the flow path direction. The vertical experiments are carried out in order to understand to what extent gravity may assist or inhibit the flow motion. The device is started up at $50 \mathrm{~W}$ in normal gravity conditions till the pseudo-steady state is reached, then the same heating cycle $(50,70$ and $100 \mathrm{~W})$ is performed at constant gravity levels. Fig. 11a shows that the PHP is working with slightly better thermal performance at $2 \mathrm{~g}$ : the average evaporator temperature can be directly compared between $1 \mathrm{~g}$ and $2 \mathrm{~g}$ for $50 \mathrm{~W}$ and $100 \mathrm{~W}$ and in both cases it is evidently lower. Furthermore the $2 \mathrm{~g}$ acceleration prevents the occurrence of local dry-outs at 100W: all the evaporator temperatures are within a narrow range till the LDC stops and the ochre temperature signal rises identically to the ground test. These results are comparable with the ones achieved with the same apparatus in the hyper-gravity phases $(1.8 \mathrm{~g})$ during the $58^{\text {th }}$ ESA Parabolic Flight Campaign [15]. Fig. $11 \mathrm{~b}$ shows that at $4 \mathrm{~g}$ acceleration strongly inhibits the fluid motion, as shown by the pressure 
diagram, at $50 \mathrm{~W}$ and many location in the evaporator undergo local fluid dry-outs and temperature increase (Thermal Crisis - zone 1).

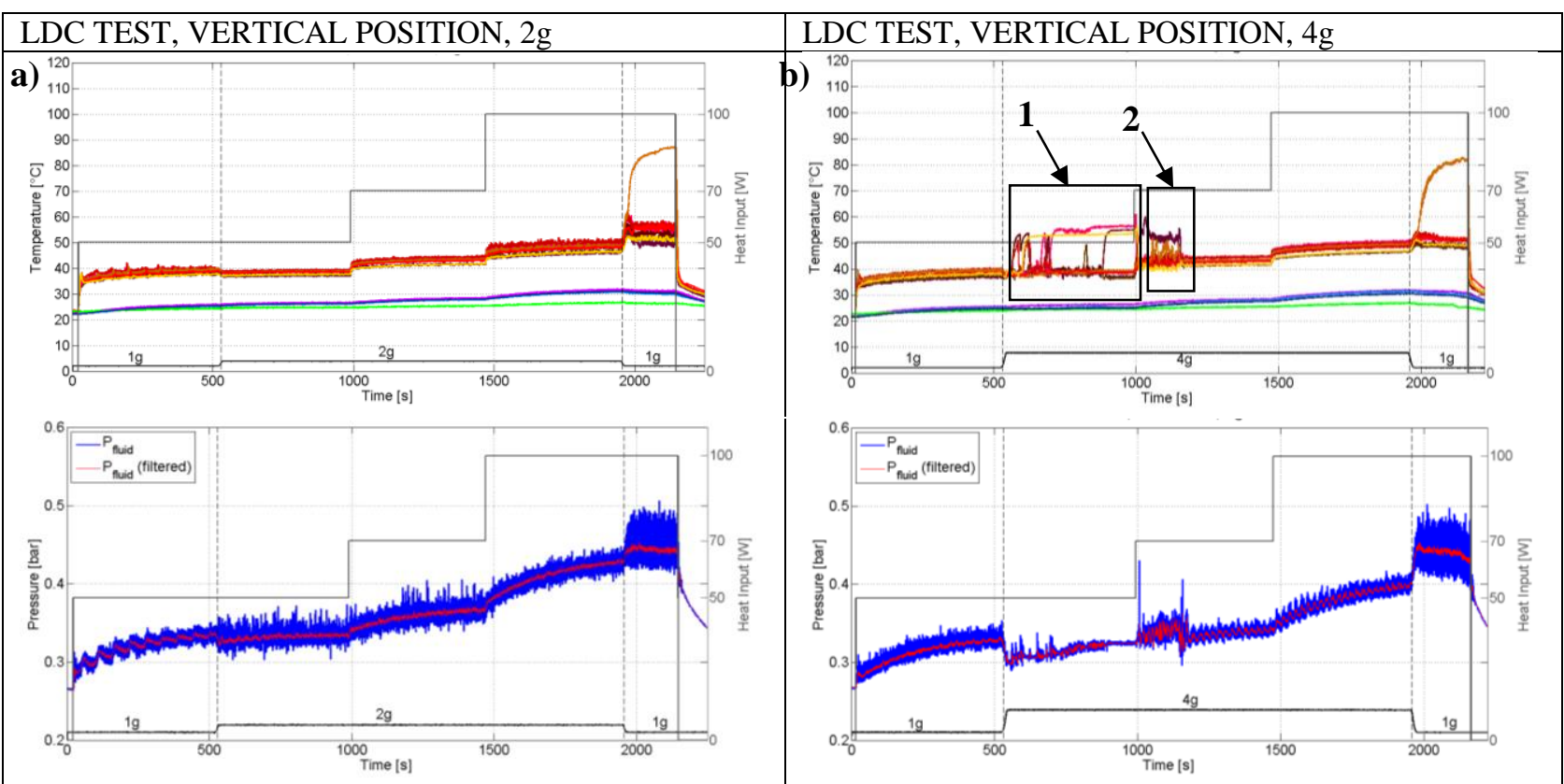

Fig. 9: Large Diameter Centrifuge experiments, a) tube wall temperatures and local fluid pressure in the vertical position at $2 \mathrm{~g}$ and different heat input levels; b) at $4 \mathrm{~g}$ and different heat input levels.

While at $70 \mathrm{~W}$ another kind of thermal instability, characterized by a more frequent stopover phenomena, resulting in large amplitude and less regular temperature and pressure oscillations, occurs (Transient Thermal Instability - zone 2). Nevertheless the system is able to resettle, after about $120 \mathrm{~s}$, and afterwards it kept a stable and efficient operation. No local thermal instabilities are detected at $100 \mathrm{~W}$ too.

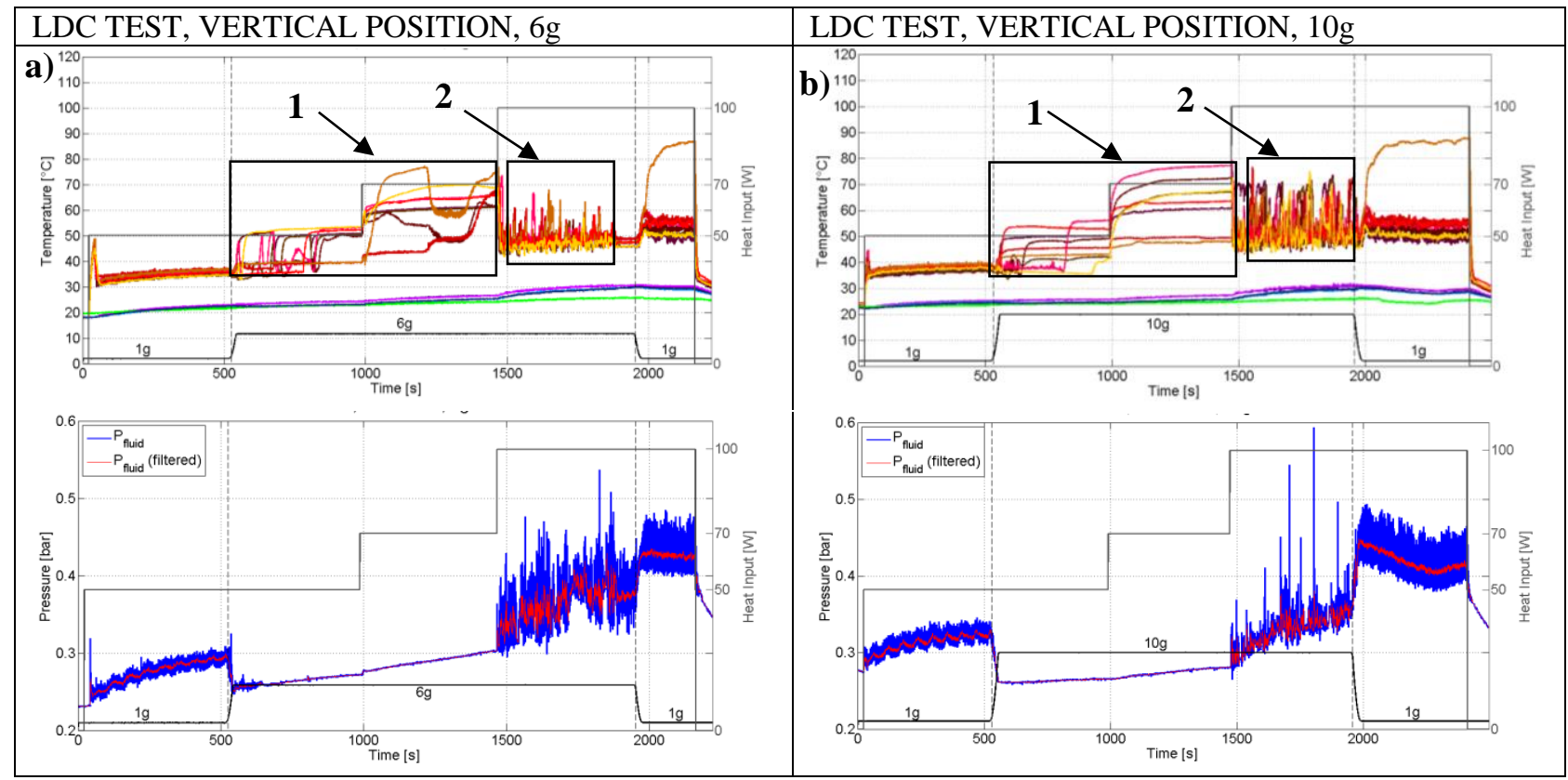

Fig. 10: Large Diameter Centrifuge experiments, a) tube wall temperatures and local fluid pressure in the vertical position at $6 \mathrm{~g}$ and different heat input levels; $\mathrm{b}$ ) at $10 \mathrm{~g}$ and different heat input levels.

At $6 \mathrm{~g}$ (Fig. 12a) the first unstable zone 1 covers a wider heat input range: not the 50W nor the $70 \mathrm{~W}$ heat input levels provide enough thermal diving force to promote a stable fluid oscillation. Only at $100 \mathrm{~W}$ the vapor expansion is strong enough to compete with the gravity acceleration, indeed after a long settlement period characterized by frequent stopovers phenomena and large amplitude temperature and pressure 
oscillations (zone 2), a stable behavior is finally reached. At 10g (Fig.12b) zone 1 covers the 50 and $70 \mathrm{~W}$ period as for the $6 \mathrm{~g}$ test but, at $100 \mathrm{~W}$, the device is not able anymore to recover from the second transient thermal instability. As for the horizontal case, additional test are performed increasing acceleration levels and keeping the heat input constant (Fig. 13). As expected, the two different thermal instabilities detected in the previous test (zones 1 and 2) occur in these experiments too. Moreover, like the horizontal mode, the performances of the PHP seem to be slightly dependent on the heating/gravity history. For example the two tests performed at the same heat input level, $70 \mathrm{~W}$, but coming from different heating/gravity paths (Fig 12a and 15a), show that the PHP respectively undergoes two different thermal instabilities at $6 \mathrm{~g}$.
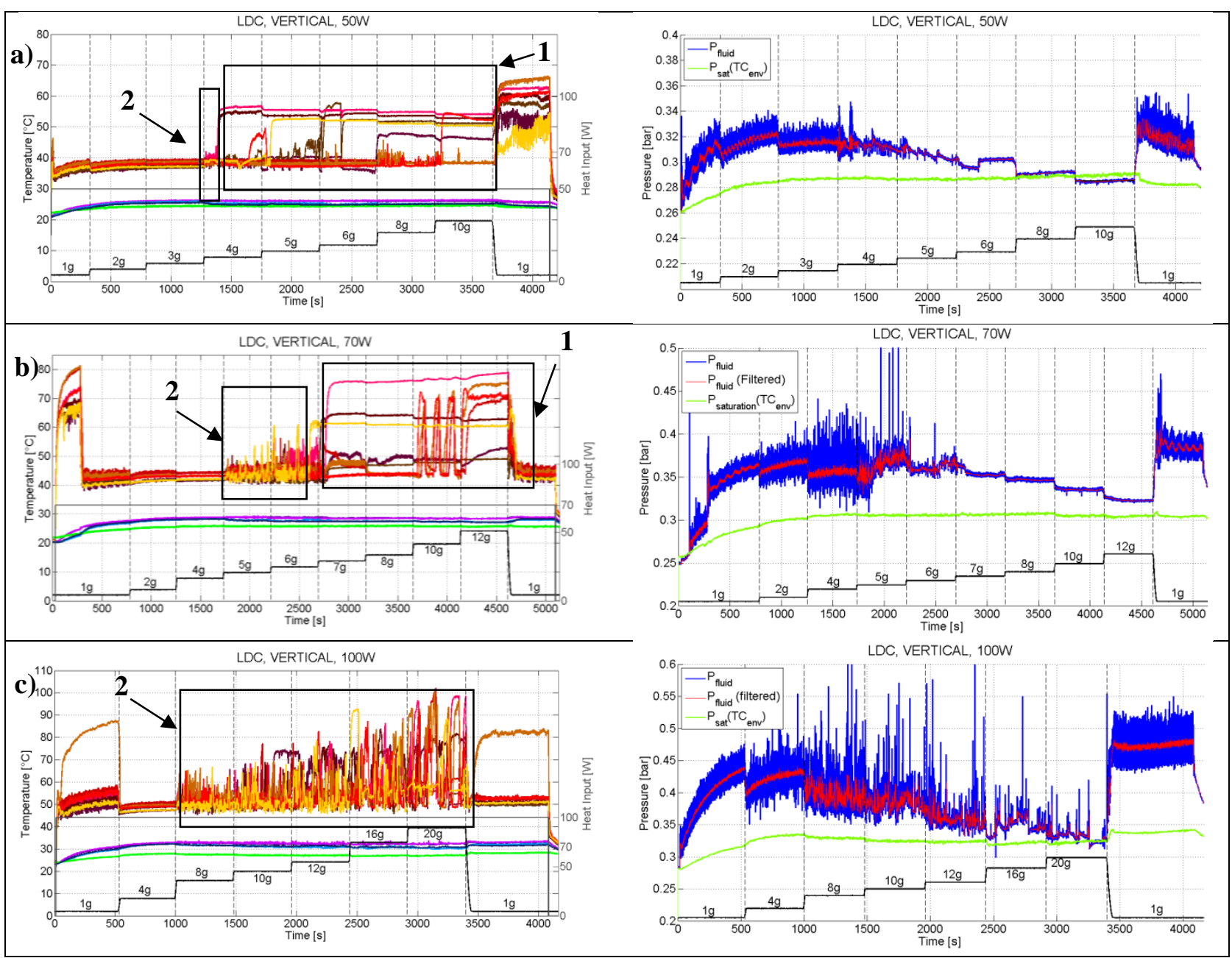

Fig. 11: Large Diameter Centrifuge experiments tube wall temperatures and local fluid pressure in the vertical position and different gravity levels at, a) $50 \mathrm{~W}$; b) $70 \mathrm{~W}$ and c) at $100 \mathrm{~W}$.

A peculiar behavior can be observed for the PHP powered with 50W: at $4 \mathrm{~g}$, after a very small period of transient thermal instability (zone 2), the device undergoes a very long thermal crisis (zone 1) and the number of dried-out channel increases with the acceleration level but, interestingly, their temperature level slightly decreases. Furthermore when the LDC stops spinning, the PHP is not able to recover the stable operation. At $70 \mathrm{~W}$, as expected, the transition between 1,2, and $4 \mathrm{~g}$ enhances the performances of the PHP. Then at 5 and $6 \mathrm{~g}$ a transient thermal instability occurs driving the PHP to a complete thermal crisis regime. This is comparable with what has been previously observed for 50W. Therefore, it seems that the transient thermal instability is, actually, a transition between a stable mode (where performances are also enhanced by the hyper-gravity) and a thermal crisis regime. Notice that at $100 \mathrm{~W}$, the transition between zone 2 and zone 1 is not so evident at least for the tested gravity levels.

Fig. 13 shows also the theoretical fluid saturation pressure calculated as a function of the environmental temperature (green line). If the fluid is not moving inside the condenser, its temperature will approach the cooling medium temperature. In terms of pressures, this means that the local fluid pressure approaches the 
saturation pressure at room temperature and the PHP is going towards worse working conditions. This is indeed what happens at increasing accelerations after the thermal crisis.

Despite internal flow visualizations could not be performed during the present experimental campaign a possible physical explanation of the above phenomenon may be deduced on the basis of the fluid local pressure. When the PHP is working in the vertical bottom heated mode, the gravity force helps the liquid phase to flow from the condenser section to the evaporator zone. This alternation of evaporation and condensation assists the motion of the fluid inside the capillary tube and, at normal gravity, an alternation of liquid slug and vapor plugs could be achieved. A small increase of the gravity improves the performances of the PHP while, if gravity increases too much, the liquid phase is forced to flow in the evaporator section but the heating power is not any more sufficient to push it up in the condenser. This leads to the formation of a liquid storage in the bottom of the device and a vapor amass in the top section. Since heating power is provided in the evaporator, bubbles may be still generated within the liquid storage causing a local and more chaotic fluid motion observed in the pressure signal that is oscillating in a less regular way with respect to the stable operation. Temperature in the evaporator section also undergoes unstable and incoherent oscillations (zones 2, Transient Thermal Instability). The bubbles generated by the boiling process, however, are inhibited by a further increase of the gravity acceleration until the system reaches the overall thermal crisis (zones 1). In this case pressure oscillations are damped and moreover, when gravity increases, the pressure signal shows a step reduction comparable with the gravity step enhancement. Finally, it is assumed that at very high gravity levels bubbles collapse as soon as they are formed because of the very high hydraulic pressure. In this condition, the system reach an equilibrium state, and saturation pressure can be measured in the top vapor.

\section{CONCLUSIONS}

A multi-turn closed loop PHP with standard 2D geometry has been experimentally investigated on ground and in hyper-gravity conditions on the Large Diameter Centrifuge at ESA-ESTEC. The temporal trend of the wall temperature in several locations (evaporator and condenser) and the local fluid pressure signal show that the PHP thermal response is strongly and rapidly affected by the variation of the gravity field. In particular the following salient points raise:

- On ground, vertical and horizontal orientations show very different behaviors confirming that, in a perfect 2D layout with a relatively high number of channels, the device orientation with respect to gravity still plays an important effect on the PHP thermal behavior.

- For the horizontal orientation, fluid stratification and the consequent thermal crisis should occur between $2 \mathrm{~g}$ and $3 \mathrm{~g}$, on the only basis of the Bond Number Criterion. Instead experiments show that the device operation stops at different acceleration levels depending also on the heat input power level and on the heating/gravity history.

- During the vertical operation gravity either assists or inhibits the flow motion depending on the combined effect of gravity and heat input level.

- The vertical PHP thermal performance is slightly enhanced by the lower hyper-gravity levels (up to $3 \mathrm{~g}$ at $50 \mathrm{~W}$, up to $4 \mathrm{~g}$ at $70 \mathrm{~W}$ and up to $6 \mathrm{~g}$ at $100 \mathrm{~W}$ ).

- Two different local thermal crisis affect the PHP thermal behavior in the vertical position: if the fluid pumping forces resulting from the heating power can compete with the acceleration forces the system only undergoes local frequent stopover phenomena (Transient Thermal Instability), while increasing with the acceleration level, the pumping forces are completely damped in some channels, the relative wall temperature increases and settles to a higher level (Thermal Crisis). A possible physical explanation of this phenomenon has been provided and supported by the condenser pressure diagram. 


\section{ACKNOWLEDGMENTS}

The authors acknowledge ESA's Spin You Thesis! program organizers and LIS engineers. Thanks to the Msc thesis student Corrado Roncelli for helping in the experimental assessment. The authors would like to thank Dr. Olivier Minster and Dr. Balazs Toth for their interest and support to the PHP activities and for the fruitful discussions. Finally we acknowledge all the members of the Pulsating Heat Pipe International Scientific Team, led by Prof. Marco Marengo, for their contribution in the pushing of the PHP technology for real space applications, with a particular gratitude to Prof. Sameer Khandekar, Dr. Vadim Nikolayev and Dr. Vincent Ayel.

\section{REFERENCES}

[1] Akachi, H. , Structure of a heat pipe. US Patent 4,921,041 (1990). Patent

[2] Akachi, Structure of micro-heat pipe. US Patent 5,219,020 (1993). Patent

[3] Ayel, V., Thevenot, F., Bertin, Y., Romestant, C., “ Analyse thermo-hydraulique expérimentale d'un caloduc oscillant sous champ de gravité variable”, Congrès Français de Thermique, SFT 2013, Gerardmer, du 28 au 31 mai (2013). Conference Proceedings

[4] Gu J., Kawaji M., Futamaca R., "Microgravity performance of micro pulsating heating pipe", Microgravity Science and Technology XVI-1, (2005). Journal Paper

[5] Gu J., Kawaji M., Futamaca R., "Effect of gravity on the performance on pulsating heating pipe", J. of Thermophysics and Heat Transfer, vol.18, No3, July-Sep (2004). Journal Paper

[6] Kiseev, V.M. , Zolkin, K.A., "The Influence of Acceleration on the Performance of Oscillating Heat Pipe", Proc. of the 11th International Heat Pipe Conference, Tokyo, Japan, (1999). Conference Proceedings

[7] Ma, H., Thompson, S.M. , Hathaway, A.A., Smoot, C.D., Wilson, C.A., "Experimental Investigation of a Flat-Plate Oscillating Heat Pipe During High-gravity Loading”, Processing of the ASME 2011 International Mechanical Engineering Congress \& Exposition IMECE2011-64821, Denver, Colorado, USA 11-17 November (2011). Conference Proceedings

[8] Van Es, J., Woering, A.A., "High-acceleration performance of the Flat Swinging Heat Pipe", NLR-TP-2000-265, (2000).

[9] Delil, A.A.M., "Microgravity two-phase and heat transfert", NLR-TP-99429, (2001). Internal report

[10] Delil, A.A.M., "Pulsating and oscillating heat transfer device in acceleration environment from microgravity to supergravity", NLR-TP-2001-001, (1999). Internal report

[11] Delil, A.A.M,"Scaling of Two-Phase Heat Transport Systems from Micro-Gravity to Super-Gravity Levels", Proc., Space Technology \& Applications International Forum, Albuquerque, USA, 221-229, (2001). Conference Proceedings

[12] Delil, A.A.M., "Thermal-Gravitational Modeling and Scaling of Heat Transport Systems for Applications in Different Gravity Environments: Super-Gravity Levels \& Oscillating Heat Transfer Devices", NLR-TP-2000-213, (2000). Internal report

[13] Van Loon, J. J. W. A., Krouse J., Kunha, U., Goncalves, J., Almeida, H., Schiller, P., "The Large Diameter Centrifuge, LDC, for Life and Physical Sciences and Technology", Proc. of the "Life in Space for Life on Earth Symposium". Angers, France 2227 June 2008 (ESA SP-663, December 2008). Conference Proceedings.

[14] Baldassari C., Marengo M.," Flow Boiling in Microchannels and Microgravity" Progress in Energy and Combustion Science Volume 39, Issue 1, February 2013, Pages 1-36. Journal Paper.

[15] Araneo L., Filippeschi S., Mameli M., Marelli L., Testa R., Manno V., Marengo M. "Thermo-Hydraulics Characterization Of A Pulsating Heat Pipe in a Variable Gravity Regime", 8th International Topical Team Workshop on Two-Phase Systems For Ground And Space Applications. Bremen, Germany, September 16-19, 2013. Conference Proceedings. 